\section{Evolution of oncogenes}

\section{From c-abl to v- $a b l$}

The discovery that the oncogenes of retroviruses are derived from cellular genes has led to a search for differences between the viral gene and its cellular counterpart that might explain the oncogenic activity of the former. Two oncogenes, the abl and the src genes, have recently come under such scrutiny. The src gene will be the subject of a later article: here, Jean Yin Jen Wang discusses the abl gene.

ABELSON murine leukaemia virus (A-MuLV) is an acute oncogenic retrovirus which induces lymphosarcoma in mice and transforms fibroblasts and pre-B lymphocytes in culture. The genome of A-MuLV contains a sequence derived from the mouse genome: by convention, this host-derived sequence is called $\mathrm{v}-a b l$ and the corresponding cellular gene (proto-oncogene) is called c-abl. The genome of A-MuLV is a hybrid of $\mathrm{v}-a b l$ and Moloney leukaemia virus (MLV) in which the $\mathrm{v}-a b l$-coding sequence is linked to a portion of the MLV genome and the expression of this coding sequence is controlled by the promoterenhancer of MLV. An obvious approach to the study of how $\mathrm{v}-a b l$ induces oncogenic transformation is to compare it with the $c-a b l$ gene whose product is a normal constituent of the cell.

MLV has a strong promoter: viral RNA produced from an integrated viral genome constitutes about 1 per cent of the total poly(A)-containing RNA of the cells in transformed fibroblasts or pre-B lymphocytes ${ }^{1}$. At the same time, cells transformed by A-MuLV express c- $a b l$ RNA from the normal cellular gene at levels 15 times lower than those of the $\mathrm{v}-a b l$ product; $c-a b l$ expression does not seem to be affected by the production of the viral RNA ${ }^{1}$. It has not yet been determined however whether the increased expression of the viral sequence contributes to the oncogenicity of $\mathrm{v}-a b l$.

The mouse $c-a b l$ gene is split into at least 10 exons which are present in $\mathrm{v}-a b l$ as a correctly spliced unit ${ }^{2}$. Thus, v-abl can be viewed as a cDNA copy of the c-abl RNA. A cDNA clone containing 1.5 kilobases (kb) of the 3 ' end of a $c-a b l$ RNA has been obtained which shows that c-abl RNA extends beyond the $3^{\prime}$ end of $v-a b l$ by 850 base pairs (bp). The difference between $\mathrm{v}-a b l$ and $c-a b l$ RNA has been analysed by primer extension experiments whose results indicate that approximately $350 \mathrm{bp}$ on the $5^{\prime}$ end of the c-abl RNA is also absent from the A-MuLV genome ${ }^{1}$. Therefore, $\mathrm{v}-a b l$ is an internal fragment of the $c-a b l$ RNA. The nucleotide sequence of the $c-a b l$ DNA shows that $\mathrm{v}-a b l$ begins within an exon and some coding sequences on the $5^{\prime}$ end of the $c-a b l$ gene are deleted from the A-MuLV genome ${ }^{2}$. There is moreover at least one base mutation in $\mathrm{v}-a b l$ which results in an amino acid substitution. Thus $v-a b l$ differs from $c-a b l$ both by deletions and by point mutations.

The hybrid genome of A-MuLV gives rise to a fusion protein in which the first 30,000 molecular weight is derived from the MLV gag gene and the remaining
130,000 molecular weight from v-abl. Expression of the $\mathrm{v}$ - $a b l$-coding sequence alone in Escherichia coli has shown that it encodes a tyrosine-specific protein kinase which phosphorylates itself and bacterial proteins $^{3}$. The A-MuLV-transformed cells have been shown to contain high levels of phosphotyrosine which can therefore be attributed to the tyrosine kinase activity of the $v-a b l$-encoded protein. Not all the $v$ - $a b l$-coding sequence is necessary to encode this kinase - expression of different segments of the $\mathrm{v}$ - $a b l$-coding sequence in bacteria shows that $1.2 \mathrm{~kb}$ on the $5^{\prime}$ end of

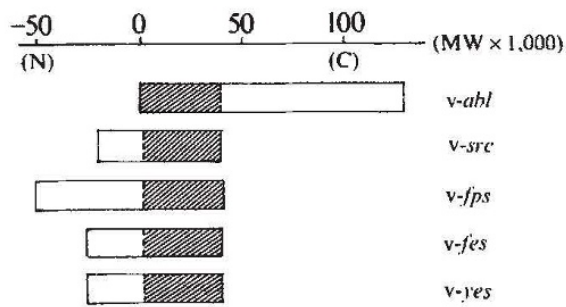

Amino acid homology of five tyrosine kinases.

The nucleotide sequence of $v-a b l$ has been determined ${ }^{5}$. Although $\mathrm{v}-a b l$ and $\mathrm{v}$-src do not cross-hybridize, the amino acid sequence derived from the $1.2-\mathrm{kb}$ kinase-coding region of $\mathrm{v}-a b l$ is about 50 per cent homologous to the C-terminal region of $\mathrm{pp} 60^{\text {src }}$, which is also a tyrosine kinase. Three other tyrosine kinases encloded by v-yes, v-fps and v-fes share this amino acid homology as well ${ }^{6-8}$. A schematic diagram of the homologous region of these five tyrosine kinases is shown in the figure. Because the coding sequence for this homologous region is sufficient for the production of an active kinase, this conserved protein sequence can be viewed as a tyrosine kinase domain which is connected to other different sequences in each of the five proteins.

The MLV gag sequence is not necessary for the tyrosine kinase activity of the A-MuLV fusion protein and 27,000 out of the 30,000 -molecular weight gag protein can be eliminated without any effect on the ability of A-MuLV to transform fibroblast ${ }^{9}$. However, while the 160,000 -molecular weight fusion protein can transform pre-B lymphocytes present in bone marrow, the gag 130,000 -molecular weight protein fails to induce bone marrow transformation in culture. To demonstrate the necessity of gag in the transformation of lymphocytes, R. Prywes constructed another set of A-MuLV variants which either contain 30,000 -molecular weight $\left(\mathrm{gag}^{+}\right)$or $\mathrm{v}-a b l$ is sufficient ${ }^{4}$. 3,000-molecular weight ( $\left.\mathrm{gag}^{-}\right)$of $\mathrm{gag}$ sequence linked to the first $2.0 \mathrm{~kb}$ of $\mathrm{v}-a b l$. Clones of both variant DNA can transform NIH 3T3 cells with equal efficiency and viral stocks containing these two variant genomes have comparable focus-forming titres. When these viral stocks were given to bone marrow cells in culture, the $\mathrm{gag}^{+}$ virus produced transformed colonies but the gag $^{-}$one did not. These results show that while 90 per cent of the MLV gag sequence in $\mathrm{A}-\mathrm{MuLV}$ is not necessary for the transformation of $3 \mathrm{~T} 3$ cells, 10 per cent of it is not enough for lymphoid transformation. Strictly speaking, therefore, the oncogene of A-MuLV for pre-B lymphocytes is the $g a g / \mathrm{v}-a b l$ hybrid rather than $v-a b l$ itself - whereas v- $a b l$ alone is probably sufficient for the transformation of NIH 3T3 fibroblasts.

In fact, not all of $v-a b l$ is required for oncogenicity. As described above, the $\mathrm{gag}^{+}$variant containing only the $5^{\prime} 2.0 \mathrm{~kb}$ of $\mathrm{v}-a b l$ can transform both fibroblasts and bone marrow cells. The smallest A-MuLV with transforming ability contains $100 \mathrm{bp}$ of gag (molecular weight 3,000) and only the $5^{\prime} 1.2 \mathrm{~kb}$, that is the kinase-coding region, of $\mathrm{v}-a b l^{9}$. It seems that all it takes is the $\mathrm{v}-a b l$ tyrosine kinase to transform 3T3 cells.

Because the $\mathrm{v}$ - $a b l$-coding sequence is derived from $c-a b l$, it is reasonable to assume that $\mathrm{c}-a b l$-encoded protein is also a tyrosine kinase with another polypeptide sequence on its $\mathrm{C}$-terminal end. Although the function of that $\mathrm{C}$-terminal region of the $c-a b l$ protein is unknown, it must be physiologically significant. However, that function is totally dispensible for the transforming activity of $\mathrm{v}-a b l$.

Since the normal physiological function of $c-a b l$ is not involved in the oncogenicity of $\mathrm{v}-a b l$, the tyrosine kinase activity may cause transformation by two different mechanisms. The v-abl kinase may have lost its specificity due to its mutations or its higher expression so that it phosphorylates protein(s) which should not be so modified and the mistake(s) triggers transformation. Or, the v-abl kinase phosphorylates its physiological substrate(s) (those of the $c-a b l$ kinase) in an unregulated fashion due to its mutations and this results in transformation. Although we still do not understand the mechanism of transformation, the comparison between $\mathrm{v}-a b l$ and $\mathrm{c}-a b l$ has clearly indicated the direction to be taken towards this end: to study the tyrosine-specific protein kinase.

Jean Yin Jen Wang is at the Department of Biology, Massachusetts Institute of Technology, Cambridge, Massachusetts, 02139.

\footnotetext{
1. Wang, J.Y.J. \& Baltimore, D. Molec. cell. Biol. (in the press)

. Wang, J.Y.J. et al. (in preparation)

3. Wang, J.Y.J., Queen, C. \& Baltimore, D. J. biol. Chem, 257, 13181 (1982).

4. Wang, J.Y.J. \& Baltimore, D. (in preparation).

5. Lee, R, et al, (in preparation).

6. Kitamura, N. et al. Nature 297, 205 (1982)

7. Shibuya, M. \& Hanafusa, H. Cell 30, 787 (1982).

8. Hempe, A. et al. Cell 30, 775 (1982)

9. Prywes, R., Foulkes, J.G., Rosenberg, N. \& Baltimore, Prywes, R., Foulk
D. (submitted).
} 